BACTERIA

\section{Mortar in the Wall}

from our Molecular Biology Correspondent

SOME of the strangest specimens in the biochemical bestiary are to be found in the bacterial cell wall, and none is more remarkable than the lipoprotein-murein from Escherichia coli, the structure of which has been described by Braun and Bosch (Proc. US Nat. Acad. Sci., 69, 970 ; 1972). The murein, or peptidoglycan, is a network of polysaccharide chains, cross-linked by short peptides, which is sandwiched between the outer wall of the cell coat and the innermost layer, the cytoplasmic membrane. Covalently attached to the murein at intervals are chains of lipoprotein, which, it has been suggested, are basic building blocks of the cell wall. The lipoprotein is released from its matrix on degradation with lysozyme, and it turns out to contain fifty-seven amino-acids.

Braun and Bosch have determined the sequence, which reveals a remarkable gene duplication phenomenon-at the N-terminus are two serine residues, which are followed by a sequence of fourteen amino-acids that is then repeated almost precisely; there are only five conservative substitutions, three of which occur at the very ends of the homologous tracts. This is followed by another substantial repeat of part of the same sequence, with residues 32-38 almost identical to 25-31. Thereafter some more short homologous tracts can be constructed if several deletions are allowed, and the chain ends in a little cluster of basic residues and a tyrosine.

A scrutiny of the sequence reveals the presence of hydrophobic residues at alternating intervals of three or four amino-acids. This is a highly characteristic arrangement for $\alpha$-helices in globular proteins, with the hydrophobic side chains lying along one side of the cylinder. That the chain is indeed $\alpha$-helical is not yet established, but is clearly to be expected, and one might suppose also that its amphiphilic character might place it at an interface between polar and non-polar media.

The point of attachment of the lipid to the murein was established from the sequence of a lipopeptide found in proteolytic digests of the lipoprotein, and turned out to be the N-terminal serine residue. The lipid, which contains no glycerol or phosphorus, and is predominantly palmitic acid, is apparently attached by way of a string of ester and amide linkages to the $\alpha$ amino group. The nature of the link between the lipoprotein and the murein was also determined. Pronase digestion, followed by partial acid hydrolysis of the murein, generated peptides containing arginine followed by lysine, which could be uniquely located at the
C-terminus of the sequence. The $\epsilon$ amino group of the C-terminal lysine is linked to one of the carboxyls of diaminopimelic acid, a species that occurs in the peptide cross-links of the murein. The diaminopimelic acid is joined by a dipeptide, glu-ala, to an $\mathrm{N}$-acetylmuramic acid residue of the polysaccharide chain. The protein chain, depending on whether it is in fact $\alpha$-helical, could project at least the greater part of the way through the cell wall, and the lipid at its extremity could then even be rooted in the membrane bilayer.

With the possibility in mind that the peptidoglycan controls the shape of the bacterial cell, Henning et al. (Europ. J. Biochem., 26, 570; 1972) have examined a mutant of $E$. coli, which, within a defined range of magnesium concentration and temperature during growth, can assume a spherical rather than the normal rod-like shape. Analysis of the murein in terms of its concentration, composition and degree of cross-linking showed no differences between spherical and rod-shaped cells, within the limits of precision, and neither was there any measurable difference in the lipoprotein fractions. It seems, therefore, that the morphological anomaly is unlikely to be related to aberrations in the murein. The outer membrane is also not a good candidate, especially because anomalous response to disruptive reagents is observed also in spheroplasts of the abnormal cells. In this case then it seems that the abnormality resides in the cytoplasmic membrane and Henning et al. provide some evidence to this effect.
It may be remarked that earlier work from Strominger's laboratory on a different organism, also capable of growing in rod or sphere forms, did show a difference in the murein: in the spheres the polysaccharide chains were shorter, and the peptide crosslinks longer, and contained glycine residues, which are not found in the rods. Henning et al. do not believe, however, that the murein can govern the overall shape, and promise evidence to support this allegation in the future. They suggest that the alteration of the murein is only one biochemical byproduct of the mutation.

Warth and Strominger (Biochemistry, 11,1389 ; 1972) have found that considerable differences exist between the mureins from spores and vegetative cells of Bacillus subtilis. Not only are a large proportion of the muramic acid residues in their $\delta$-lactam forms (giving the effect of two fused rings), but others bear $\mathrm{N}$-acetyl groups and alanine residues as substituents. The cross-linking density is low, and in consequence of this and of the pendant seryl residues, the concentration of free carboxyl groups is distinctly high. This confers on the spore surface a more polyacidic character than that of the vegetative cells. The authors' suggestion is that an increase in ionic strength will consequently elicit contraction which will also be favoured by the higher elasticity resulting from the paucity of cross-links. Contraction will be accompanied by dehydration, and this, it is thought, is in turn related to the temperature resistance that characterizes the sporulating state.

\title{
Rifampicin and Adenovirus Oncogenicity
}

THE antibiotic rifampicin has an extraordinarily wide spectrum of biological activity; it not only blocks transcription in bacteria but also inhibits the replication of trachoma agent, the replication of vaccinia and other viruses including adenoviruses, and may influence the transformation of cells by the RNA tumour viruses. Furthermore, as Toolan and Ledinko show in Nature New Biology next Wednesday (April 14), rifampicin also affects the induction of tumours in baby hamsters by adenovirus 12 .

Toolan and Ledinko, intrigued by Diggelman and Weissman's claim that rifampicin suppresses transformation of chick fibroblasts by Rous sarcoma virus, performed a set of experiments in which they injected newborn male and female hamsters with a dose of oncogenic adenovirus 12 and followed it with a series of injections of rifampicin at one of two concentrations. Control animals were injected either with the virus alone or with the drug alone or with saline. The number of animals of both sexes subsequently developing tumours and the time at which tumours first become detectable were then measured.

Toolan and Ledinko's results reveal, first, that female hamsters are significantly more susceptible to the oncogenic action of adenovirus 12 than are their male litter mates, and rifampicin if anything enhances the susceptibility of the female hamsters. By contrast, however, rifampicin, even at the lower of the two doses used in these experiments, significantly reduces the incidence of tumours in baby male hamsters inoculated with adenovirus 12 ; the tumour incidence in the treated males was 9 per cent compared with 32 per cent in the untreated controls.

Why female hamsters are more susceptible to the oncogenic action of adenovirus 12 than male hamsters and how rifampicin inhibits tumour induction in the males are at present open questions. 